\title{
"De-novo DCB-only" in complex coronary interventions and chronic total occlusion percutaneous coronary intervention
}

\section{OMihajlo Kovačić*, (DDario Dilber}

Čakovec County Hospital, Čakovec, Croatia
RECEIVED:

February 15, 2020

ACCEPTED:

February 22, 2020

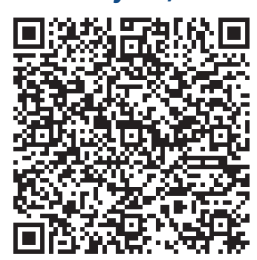

$\square$ Cardiologia Croatica 2020;15(3-4):54.
KEYWORDS: drug-coated balloon, complex percutaneous coronary interventions, chronic total occlusion. CITATION: Cardiol Croat. 2020;15(3-4):54-6. | https://doi.org/10.15836/ccar2020.54

*ADDRESS FOR CORRESPONDENCE: Mihajlo Kovačić, Županijska bolnica Čakovec, I. G. Kovačića 1E, HR-40000 Čakovec, Croatia. / Phone: +385-98-266-774 / E-mail: mihajlo1983@gmail.com

ORCID: Mihajlo Kovačić, https://orcid.org/0000-0002-2577-9474 • Dario Dilber, http://orcid.org/0000-0002-0062-4708

|IIIIIIIIIIIIIIIIIIIIIIIIIIIIIIIIIIIIIIIIIIIIIIIIIIIIIIIIIIIIIIIIIIIIIIIIIIIIIIIIIIIIIIIIIIIIIIIIIIIIIIIIIIIIIIIII

Introduction: Drug-coated balloon (DCB, PACCOCATH ${ }^{\circledR}$ technology) is recognized from 2014 ESC Guidelines on myocardial revascularization in treatment of DES/BMS ISR (IA recommendation) ${ }^{1}$ and its indications are expanding. DCB in "de-novo" lesions is validated mostly in Small Vessel Disease (PEPCAD I, PICCOLETO, BASKET-SMALL 2, International SVD Register). Information on Large Vessel Disease are scarce and based on preparation of the lesion with DCB followed with BMS implantation: PEPCAD IV and OCTOPUS I, PEPCAD V (Bifurcations), DEBAMI (Acute Myocardial Infarction) and PEPCAD CTO, but "de-novo DCB-only" concept is still practically "off label". ${ }^{2}$ Available conclusions on "de-novo DCB-only" concept can be drawn from the World-wide "all comer" Registry and single center studies such as Potsdam Heart Center, trials such as OCTOPUS II (Stabile CAD), DCB Bifurcation Study (Side Branch Treatment), study on Primary Percutaneous Coronary Intervention ${ }^{3}$ and works of Kleber and coworkers which studied Late Lumen Enlargement in DCB-only concept, but again, mainly in small vessels. "De-novo DCB-only" in complex coronary interventions is practically "off label", especially in CTO PCI. In this paper we present a complex PCI based on "de-novo DCB-only" concept and a novel approach to CTO PCI based on this method.

Case 1: 85-years-old female patient was admitted due to acute coronary syndrome. Diffuse coronary disease was found, occlusion of LCX, CTO of ostial PD. Heart team decided for PCI. Procedure was done via bilateral radial approach with support of dual lumen microcatheter, and AWE technique. Final angioplasty (Figure 1) was done with DCB 3.0x30 Sequent Please NEO (B. Braun).

Case 2: 78-years-old male patient with multiple previous PCIs was admitted for elective PCI of in-stent restenosis CTO of OM1. Previously, ostial lesion of OM2 was also p treated with DCB. CTO procedure was done via right transradial approach with support of microcatheter and AWE technique. Final procedure (Figure 2) was done with 2 DCBs covering ostial and distal OM1, and 1 DES covering fractured microcatheter tip.

Case 3: 78-years-old male patient with multiple comorbidities and previously done CABG was admitted for elective PCI after verification of vein grafts degeneration. CTO PCI of LAD (Figure 3) was done with AWE technique and finalized with angioplasty with DCB 2.0x30.
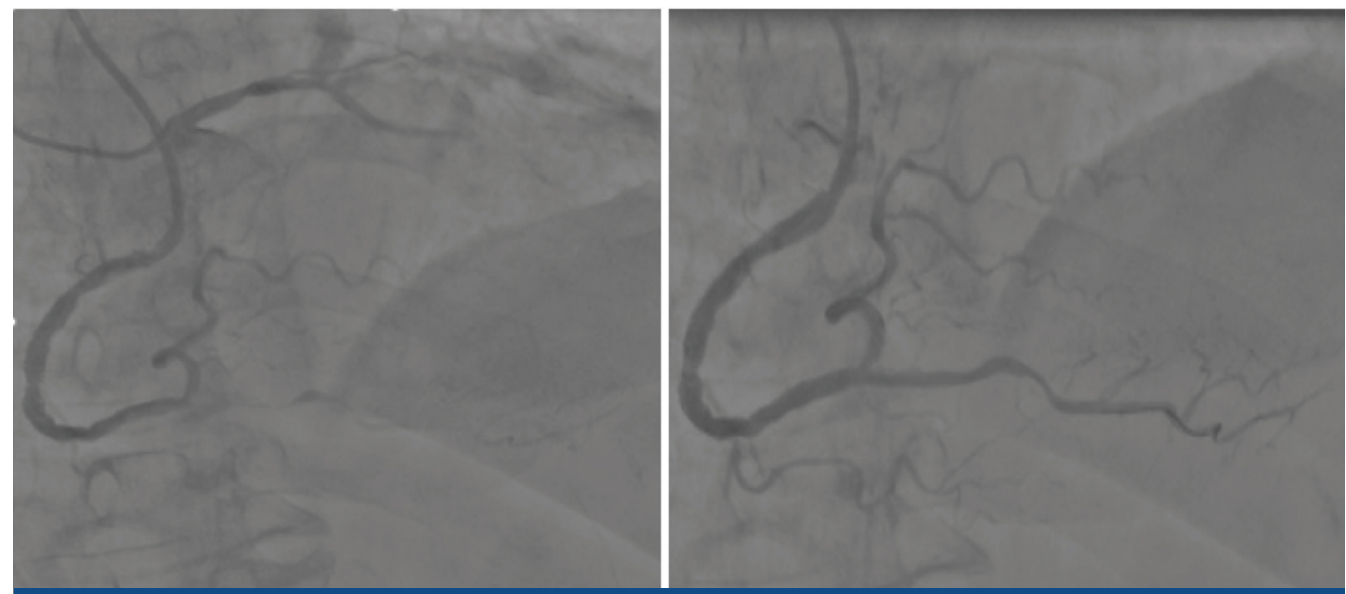

FIGURE 1. Angiogram before and after angioplasty with drug-coated balloon. 

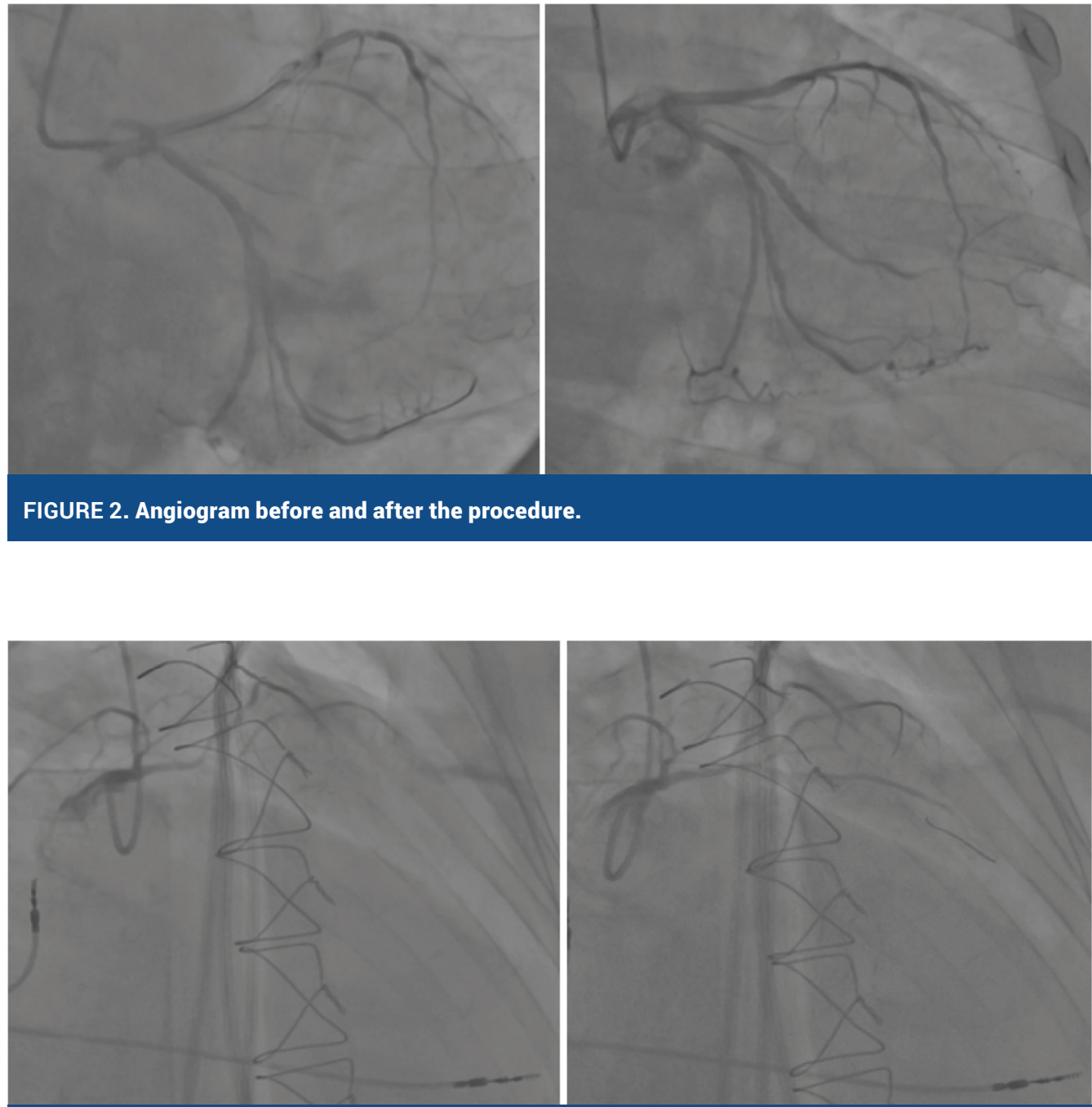

FIGURE 3. Angiogram before and after angioplasty with drug-coated balloon.

Case 4: 85-years-old male patient was admitted for elective PCI CTO of RCA. Procedure was done via left transradial approach and single guiding catheter. AWE technique was done, after which CTO body was treated with DCBs $2.0 \times 25$ and $2.5 \times 25$ and mid segment of the RCA was treated with DES 2.75x33 (Figure 4)
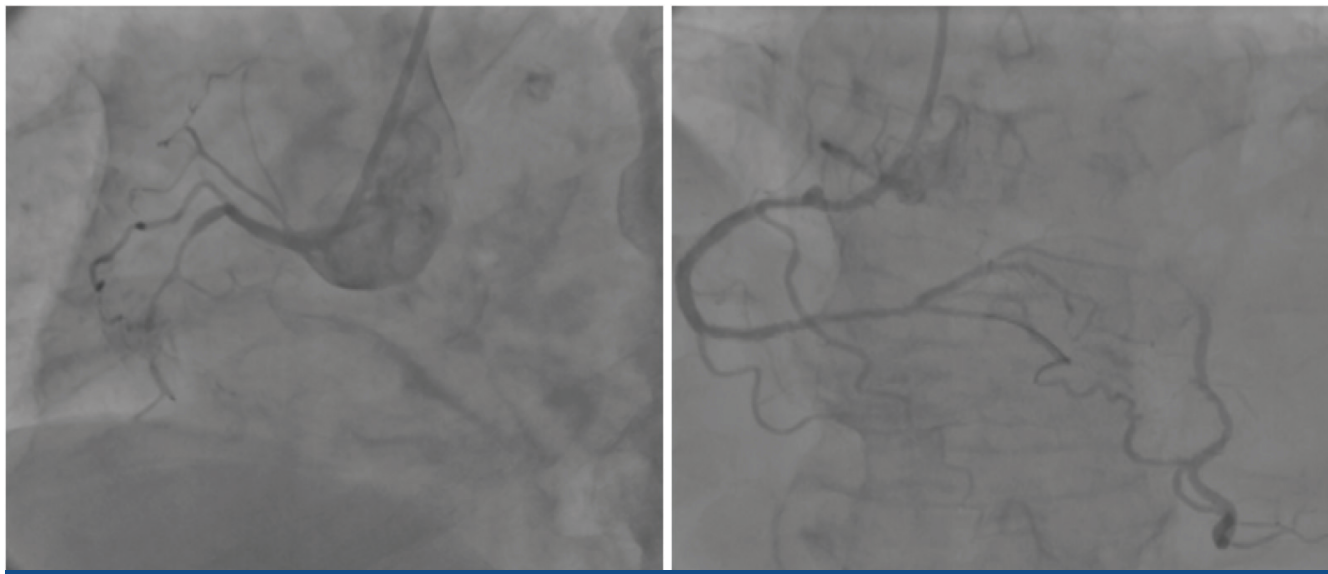

FIGURE 4. Angiogram before and after the procedure. 
“De-novo DCB-only" in complex coronary interventions and chronic total occlusion percutaneous coronary intervention

Case 5: 78-years-old female patient was admitted for elective PCI CTO of LAD. Procedure was done with AWE technique, and finalized with two DCBs, 2.0x30 in mid and 2.5x30 in distal segment of the LAD, and 1 DES at the bifurcation of LAD-D1 (Figure 5).
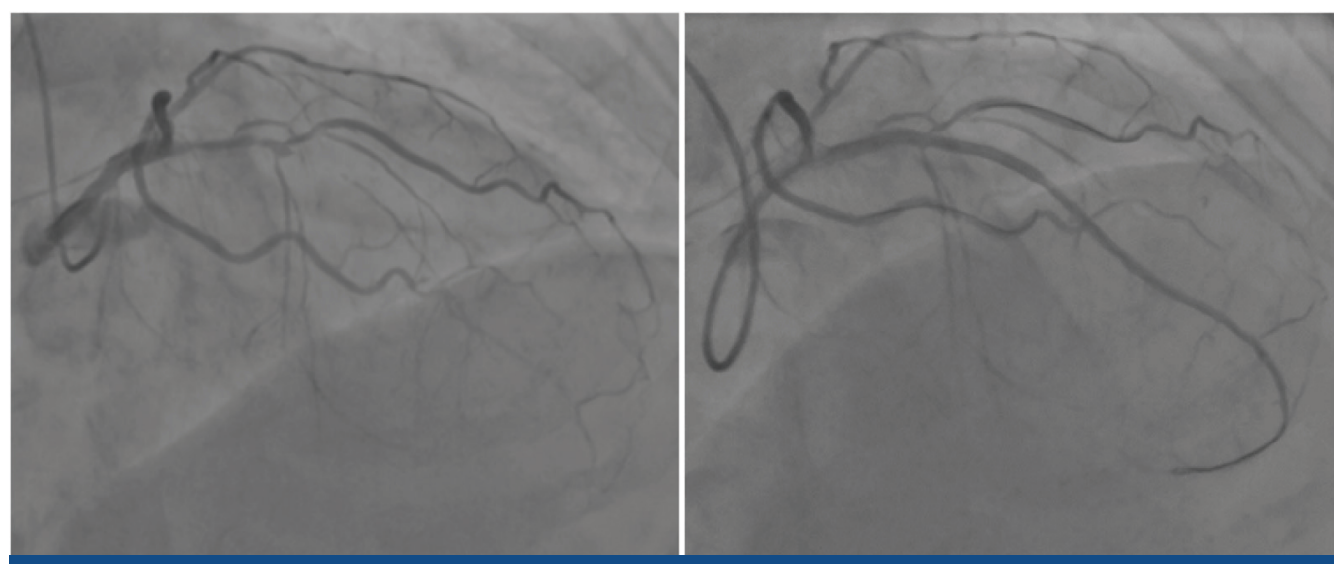

FIGURE 5. Angiogram before and after the procedure.

Conclusion: "De-novo DCB-only" concept in complex coronary interventions in chronic coronary syndrome as well as in the setting of primary PCI shows promising results, especially for ostial lesions and by simplifying bifurcations techniques without compromising final result. Concept of de-novo DCB-only CTO procedure depicted here, with "true-to-true crossing" and optimal lesion preparation, shows excellent results, but follow-up and further studies are warranted.

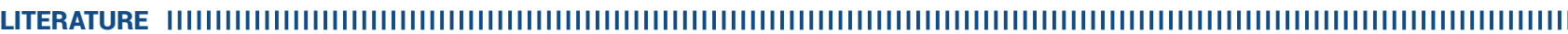

1. Authors/Task Force members, Windecker S, Kolh P. Alfonso F, Collet JP. Cremer J, Falk V, et al. 2014 ESC/EACTS Guidelines on myocardial revascularization: The Task Force on Myocardial Revascularization of the European Society of Cardiology (ESC) and the European Association for Cardio-Thoracic Surgery (EACTS)Developed with the special contribution of the European Association of Percutaneous Cardiovascular Interventions (EAPCI). Eur Heart J. 2014 0ct 1;35(37):2541-619. https://doi.org/10.1093/eurheartj/ehu278

2. Gobić D, Tomulić V, Lulić D, Židan D, Brusich S, Jakljević T, et al. Drug-Coated Balloon Versus Drug-Eluting Stent in Primary Percutaneous Coronary Intervention: A Feasibility Study. Am J Med Sci. 2017 Dec;354(6):553-560. https://doi.org/10.1016/j.amjms.2017.07.005

3. Wickramarachchi U, Corballis NH, Maart CA, Gilbert TJ, Eccleshall SC. 24 Drug coated balloon-only angioplasty in chronic total occlusions, a UK single centre experience Heart 2017;103:A11. https://doi.org/10.1136/heartjnl-2017-BCIS.24 